\title{
Empirisch-analytische Friedens- und Konfliktforschung in Deutschland: Ein Kommentar zur Evaluation durch den Wissenschaftsrat
}

\author{
Constantin Ruhe $\mathbb{D} \cdot$ Julia Leib $\mathbb{D} \cdot$ Nils B. Weidmann • Margit Bussmann
}

Eingegangen: 17. September 2020 / Überarbeitet: 15. Oktober 2020 / Angenommen: 20. Oktober 2020 / Online publiziert: 6 . November 2020

(C) Der/die Autor(en) 2020

Zusammenfassung Dieser Beitrag reflektiert und ergänzt die aktuelle Diskussion über die Empfehlungen des Wissenschaftsrats zur Weiterentwicklung der Friedensund Konfliktforschung. Wir richten dabei den Blick auf die vom Wissenschaftsrat attestierten Schwachstellen im Bereich empirisch-analytischer Methoden und erläutern ihre Auswirkungen auf Interdisziplinarität, Internationalität und Politikberatung der deutschen Friedens- und Konfliktforschung. Wir argumentieren, unter Verweis auf den Bericht des Wissenschaftsrats, dass eine breitere Methodenausbildung und -kenntnis von großer Bedeutung für interdisziplinäre und internationale Zusammenarbeit, aber auch für die Politikberatung ist. Zukünftige Initiativen innerhalb der Friedens- und Konfliktforschung sollten die Methodenvielfalt des Forschungsbereichs angemessen berücksichtigen und einen besonderen Fokus auf die Ausbildung im Bereich empirisch-analytischer Methoden legen, um das Forschungsfeld in diesem Bereich zu stärken. Unser Beitrag entspringt einer Diskussion innerhalb des

C. Ruhe $(\bowtie)$

Institut für Politikwissenschaft, Fachbereich Gesellschaftswissenschaften, Goethe-Universität

Frankfurt, Theodor-W.-Adorno-Platz 6, 60323 Frankfurt am Main, Deutschland

E-Mail: ruhe@soz.uni-frankfurt.de

J. Leib

Wirtschafts- und Sozialwissenschaftliche Fakultät, Universität Potsdam,

August-Bebel-Straße 89, 14482 Potsdam, Deutschland

E-Mail: leib@uni-potsdam.de

\section{N. B. Weidmann}

Fachbereich Politik- und Verwaltungswissenschaft, Universität Konstanz, Universitätsstr. 10, 78457 Konstanz, Deutschland

E-Mail: nils.weidmann@uni-konstanz.de

\section{Bussmann}

Institut für Politik- und Kommunikationswissenschaft, Universität Greifswald,

Ernst-Lohmeyer-Platz 3, 17489 Greifswald, Deutschland

E-Mail: margit.bussmann@uni-greifswald.de 
Arbeitskreises „Empirische Methoden der Friedens- und Konfliktforschung“ der Arbeitsgemeinschaft Friedens- und Konfliktforschung.

Schlüsselwörter Forschungsmethoden · Wissenschaftsrat · Interdisziplinarität · Internationalisierung $\cdot$ Politikberatung $\cdot$ Nachwuchsförderung $\cdot$ Methodenpluralismus

\section{Empirical-analytical peace and conflict research in Germany: a comment on the evaluation by the german science council}

Abstract This article reflects on and adds to the ongoing discussion of the German Science Council's recommendations for the further development of peace and conflict research. We focus on the gaps in empirical-analytical research methodology identified by the German Science Council and elaborate how they affect interdisciplinary cooperation, international visibility and policy-oriented research within the German peace and conflict research community. We follow the analysis of the Science Council's report and argue that a diversified training in as well as knowledge of empirical research methodology is of central importance for interdisciplinary and international cooperation as well as comprehensive policy-oriented research. Future initiatives within the peace and conflict research community should strive to reflect the methodological diversity of our research community and put a special emphasis on training in empirical-analytical research methodology, in order to strengthen the methodological expertise in this realm. Our article originates from a discussion within the working group on "Empirical Research Methods in Peace and Conflict Research" of the German Association for Peace and Conflict Studies' (Arbeitsgemeinschaft für Friedens- und Konfliktforschung e.V.-AFK).

Keywords Research methods - German science council · Interdisciplinary research · International cooperation · Policy recommendations · Promoting young researchers $\cdot$ Methodological pluralism

Der Wissenschaftsrat hat mit der Begutachtung der Friedens- und Konfliktforschung im vergangenen Jahr eine außergewöhnlich umfassende Evaluation eines gesamten Forschungsbereichs vorgelegt, an der in unserem Forschungsfeld ,im deutschsprachigen Raum in Zukunft niemand vorbeikommen wird“ (Gehring und Rubner 2020, S. 177). Es ist erfreulich, dass der Wissenschaftsrat den Wert der Friedens- und Konfliktforschung hervorhebt und insgesamt positiv bewertet. Die Freude über dieses Ergebnis sollte jedoch zugleich ein Anlass sein, um im Gutachten angesprochene Kritikpunkte anzugehen und Defizite durch gezielte Maßnahmen zu beheben. Die kritische Selbstreflexion hat in den bereits veröffentlichten Diskussionsbeiträgen des ZeFKo Forums begonnen (vgl. bspw. Engels und Schetter 2020; Schneckener und Held 2020; Bonacker 2020), weitere Kritikpunkte des Wissenschaftsrats sind allerdings bisher beinahe unberücksichtigt geblieben. Unser Beitrag richtet daher den Blick auf die vom Wissenschaftsrat attestierten Schwachstellen im Bereich 
empirisch-analytischer ${ }^{1}$ Methoden und ihren Auswirkungen auf Interdisziplinarität, Internationalität und Politikberatung der deutschen Friedens- und Konfliktforschung.

Die Friedens- und Konfliktforschung in Deutschland ist ein breites, interdisziplinäres Forschungsfeld. Dem Großteil der Forschenden in der Friedens- und Konfliktforschung gemein ist jedoch das thematische Interesse und die normative Motivation, die Ursachen, Formen und/oder Folgen von Konflikt und Gewalt wissenschaftlich zu erforschen, negative Auswirkungen im Idealfall zu verhindern und zu einer friedlichen Gesellschaft beizutragen. Unter diesem breiten Ziel versammelt die deutsche Friedens- und Konfliktforschung eine Vielzahl von wissenschaftlichen Perspektiven und methodischen Zugängen. Allerdings ist nach Auffassung des Wissenschaftsrats die vorhandene methodische Kompetenz in einigen Bereichen zu eng. Während beispielsweise diskursanalytische oder ethnographische Forschungsansätze verbreitet sind (vgl. Wissenschaftsrat 2019, S. 37), betont der Wissenschaftsrat die Notwendigkeit des Ausbaus von Kompetenzen in empirisch-analytischen Methoden, um - im Sinne eines Methodenpluralismus - die deutsche Friedens- und Konfliktforschung breiter aufzustellen und internationale und interdisziplinäre Kooperation zu fördern (vgl. Wissenschaftsrat 2019, S. 55).

In seinem Gutachten mahnt der Wissenschaftsrat einen stärkeren Austausch zwischen den diversen in der Friedens- und Konfliktforschung forschenden Disziplinen an. Während das Stichwort „Interdisziplinarität“ in der aktuellen Debatte bereits ein breites Echo ausgelöst hat (vgl. Engels und Schetter 2020; Niemann und Schröder 2020; Reuter et al. 2020), findet der zweite Teil der Evaluation des Wissenschaftsrats zu diesem Stichwort in der aktuellen Debatte bisher noch zu wenig Beachtung: Laut Wissenschaftsrat sind breitere Methodenkompetenzen für einen vertieften, interdisziplinären Austausch notwendig (Wissenschaftsrat 2019, S. 55). Dieses Defizit in der methodischen Ausbildung, speziell im quantitativen oder experimentellen Bereich, gerät zudem zunehmend zu einem Hindernis, um in wichtigen Debatten der internationalen Friedens- und Konfliktforschung Gehör zu finden (vgl. Wissenschaftsrat 2019, S. 37 f.).

In diesem Diskussionsbeitrag fassen wir zunächst die Schlussfolgerungen und Empfehlungen des Wissenschaftsrats zur Stärkung der Methodenkompetenz in der Friedens- und Konfliktforschung zusammen. Anschließend argumentieren wir, unter Verweis auf den Bericht des Wissenschaftsrats, dass eine breitere Methodenausbildung und -kenntnis sowie eine gestärkte empirisch-analytische Friedens- und Konfliktforschung wichtig für interdisziplinäre und internationale Zusammenarbeit ist, einen großen Einfluss auf die Politikberatung haben kann und deshalb bei zukünftigen Initiativen angemessen berücksichtigt werden sollte. Dieser Beitrag ist deshalb keine generelle Methodenkritik für das Forschungsfeld der Friedens- und Konfliktforschung in Deutschland, sondern wir folgen der Bestandsaufnahme des Wissenschaftsrats und der darin explizit identifizierten Lücken.

\footnotetext{
1 Wir verstehen empirisch-analytische Methoden als Forschungsdesigns, die empirische Phänomene wertneutral, ergebnisoffen, intersubjektiv nachvollziehbar und prinzipiell replizierbar untersuchen. Diese Forschungsdesigns können beobachtend oder experimentell sein und quantitative sowie qualitative Datenanalysen umfassen.
} 
Unser Beitrag entspringt der Diskussion innerhalb des Arbeitskreises „Empirische Methoden der Friedens- und Konfliktforschung“ der Arbeitsgemeinschaft Friedensund Konfliktforschung (AFK). Ausgehend vom Gutachten wurden die Evaluationsergebnisse in einem (virtuellen) Meeting besprochen und die wichtigsten Punkte dieses Diskussionsbeitrags herausgearbeitet. Im Anschluss entwickelte ein vierköpfiges Autor*innenteam einen ersten Entwurf dieses Beitrags, welcher vor der Einreichung wiederum von der Gesamtgruppe kommentiert wurde. ${ }^{2}$

\section{Methodenpluralität in der deutschen Friedens- und Konfliktforschung}

Im Folgenden geben wir kurz die Position des Wissenschaftsrats zur methodischen Vielfalt in der deutschen Friedens- und Konfliktforschung wieder und legen dar, welche Auswirkungen diese Einschätzung auf die interdisziplinäre Zusammenarbeit innerhalb des Forschungsgebiets, ihre internationale Sichtbarkeit und ihre Schnittstelle in die Politikberatung haben kann.

\subsection{Der Befund des Wissenschaftsrats}

Der Wissenschaftsrat gibt dem Thema Forschungsmethoden in seinem Gutachten umfassenden Raum. Er beschreibt die Relevanz einer fundierten Methodenkompetenz für die deutsche Friedens- und Konfliktforschung und konstatiert sehr deutlich bestehende Lücken in diesem Bereich: „In der deutschen Friedens- und Konfliktforschung dominiert ein qualitativer Zugang, insbesondere in der Form von Diskursanalysen und vergleichenden Fallstudien. Zunehmenden Einfluss haben in den letzten Jahren ethnographische Methoden und andere Ansätze aus den Regionalstudien gewonnen“ (Wissenschaftsrat 2019, S. 37). Damit unterscheide sich die deutsche Friedens- und Konfliktforschung deutlich vom internationalen Trend: „Die internationale Friedens- und Konfliktforschung ist gegenwärtig stark von quantitativen sozialwissenschaftlichen Zugängen geprägt. [...] An den meisten deutschen Standorten kommt der quantitativen Friedens- und Konfliktforschung nur eine untergeordnete Bedeutung zu“ (Wissenschaftsrat 2019, S. 37). Gerade diese ist jedoch - im Rahmen einer methodenpluralistischen Friedens- und Konfliktforschung - für die Analyse empirischer Muster und genereller Zusammenhänge unabdingbar.

Prinzipiell befürwortet der Wissenschaftsrat den „Anspruch der Methodenpluralität, auch in Form von mixed methods-Ansätzen“ der deutschen Friedens- und Konfliktforschung (Wissenschaftsrat 2019, S. 37). Schwierigkeiten gebe es aber bei der Umsetzung: ,Allerdings setzt dieser begrüßenswerte methodenpluralistische Anspruch fundierte Kenntnisse in einem breiten Methodenspektrum voraus. Wie das Forschungsfeld selbstkritisch feststellt, sind diese jedoch unter anderem im quantita-

\footnotetext{
2 Wir bedanken uns bei Levke Aduda, Nils-Christian Bormann, Tilman Brück, Belen Gonzalez, Felix Haas, Roos van der Haer, Tobias Ide, Carlo Koos, Daniel Lambach, Clara Neupert-Wentz, Samantha Ruppel, Adam Scharpf, Christian von Soest, Johannes Vüllers und Timothy Williams für Kommentare und Anregungen.
} 
tiven Bereich nicht immer in wünschenswertem Maße vorhanden. Dies gilt ebenso für Kompetenzen im Bereich experimenteller Methoden, die derzeit vor allem in den USA an Aufmerksamkeit gewinnen“ (Wissenschaftsrat 2019, S. 37f). Initiativen, die diese Lücke zur internationalen Friedens- und Konfliktforschung schließen möchten, bewertet der Wissenschaftsrat daher sehr positiv: ,Vor dem Hintergrund der internationalen Entwicklungen im Forschungsfeld sind Bemühungen um eine Stärkung der quantitativen Friedens- und Konfliktforschung in Deutschland zu begrüßen“ (Wissenschaftsrat 2019, S. 37).

Angesichts der kritischen Bewertung findet sich diese Thematik folglich in den fünf zentralen Empfehlungen des Wissenschaftsrats wieder: „Der Wissenschaftsrat empfiehlt den Wissenschaftlerinnen und Wissenschaftlern des Forschungsfeldes daher, insbesondere für Studierende, Promovierende und Postdocs Möglichkeiten einer standortübergreifenden vertieften Schulung im breiten Methodenspektrum der Friedens- und Konfliktforschung zu prüfen, d.h. im Bereich der qualitativen und quantitativen Methoden, insbesondere von datenintensiver Forschung und Simulationen, sowie der experimentellen Methoden“ (Wissenschaftsrat 2019, S. 60; Hervorhebungen im Original).

\subsection{Empirisch-analytische Methoden und deren Relevanz für interdisziplinäre Zusammenarbeit}

Die deutsche Friedens- und Konfliktforschung ist keine unabhängige Disziplin, sondern vielmehr ein interdisziplinäres Forschungsfeld, das geprägt ist durch eine Vielfalt theoretischer Zugänge und methodischer Ansätze (vgl. Brühl 2012). Wie der Wissenschaftsrat anmerkt, gestaltet sich diese interdisziplinäre Ausrichtung zunehmend als schwierig, da bestehende institutionelle Strukturen und Förderformate das Forschen in disziplinären und methodischen Schubladen begünstigen und zu einer disziplinären und methodischen Verengung geführt haben. Zudem arbeiten viele Forscher*innen inhaltlich zu Themen der Friedens- und Konfliktforschung, ohne dass sie ihre Ergebnisse als zu diesem Forschungsfeld zugehörig deklarieren, z. B. in den Bereichen Sozialpsychologie (vgl. Vollhardt und Cohrs 2013), Klimawissenschaften (vgl. Scheffran und Fröhlich 2017), Pädagogik (vgl. Büttner 2004) oder Wirtschaftswissenschaften (vgl. Verwimp et al. 2019). Daher mahnt der Wissenschaftsrat zu Recht einen stärkeren Austausch zwischen diversen Disziplinen sowie eine ,,dauerhafte Integration der für das Forschungsfeld erforderlichen Vielfalt theoretischer Zugänge und methodischer Ansätze“ an (Wissenschaftsrat 2019, S. 46). Nur so ließe sich die Vielzahl an interdisziplinären Fragestellungen angemessen bearbeiten. Hierfür wären jedoch aktive Outreach-Programme, beispielsweise durch die AFK, nötig, um Forscher*innen für gemeinsame Projekte zu vernetzen.

Eine nachhaltige Integration und Zusammenarbeit verschiedener Disziplinen innerhalb der Friedens- und Konfliktforschung setzt jedoch eine Kommunikationsfähigkeit auf methodischer Ebene und, unter anderem, auch ein breites Verständnis empirischer Methoden voraus (vgl. Schneckener und Held 2020, S. 164). Als Beispiele seien hier das Network of European Peace Scientists sowie das Households in Conflict Network genannt, in dem sich jeweils Politikwissenschaftler*innen, Ökonom*innen, Soziolog*innen und Vertreter*innen weiterer Disziplinen gerade auf- 
grund einer kompatiblen Methodenkompetenz zu Fragen der Friedens- und Konfliktforschung austauschen. In Anlehnung an die Empfehlungen des Wissenschaftsrates argumentieren wir daher, dass Methodenpluralität und eine breite Methodenkompetenz eine notwendige Grundlage für Interdisziplinarität und die geforderte Perspektivenerweiterung sind. Die vom Wissenschaftsrat im Bereich der Friedens- und Konfliktforschung angemahnte interdisziplinäre Zusammenarbeit zwischen Sozialund Geisteswissenschaften sowie stark empirisch orientierten Disziplinen, wie der Psychologie, Ökonomie, Informatik und den Naturwissenschaften setzt prinzipiell voraus, dass die daran beteiligten Forscher*innen über ein grundlegendes Verständnis empirisch-analytischer Forschung und elementare Methodenkenntnisse im qualitativen sowie quantitativen Bereich verfügen. Allerdings möchten wir auch betonen, dass nicht jede*r Wissenschaftler*in alle Methoden als Anwender*in beherrschen muss. Auf der Basis gegenseitigen Verständnisses und Respekts sowie einer wechselseitigen wissenschaftlichen Neugier sollten aber die verschiedenen Methoden und Ansätze in der Summe einander bereichern. Gerade in großen Forschungsverbünden und Clustern stellt die individuelle methodische Herangehensweise jedoch häufig ein Kommunikationsproblem dar und erschwert die interdisziplinäre Zusammenarbeit. Dies betrifft auch den Austausch zwischen evidenzbasierter und normativer Friedens- und Konfliktforschung in interdisziplinären Projekten, denn eine valide Beschreibung von empirischen Zusammenhängen ist meist die Voraussetzung für deren normative Beurteilungen. Gleichzeitig werfen normative Ansätze Schlaglichter auf wichtige Fragestellungen, die auch empirisch-analytische Forschung bereichern. Hier braucht es in der Friedens- und Konfliktforschung jedoch mehr Austausch und gegenseitiges Verständnis, um die Arbeit in gemeinsamen Projekten zu fördern und das Denken in dichotomen, getrennten Wissenschaftsvorstellungen zu reduzieren.

\subsection{Empirisch-analytische Methoden und deren Relevanz für Internationalisierung}

Methodenpluralismus und eine gestärkte empirisch-analytische Methodenausbildung sind zudem notwendig, um zentrale Empfehlungen des Wissenschaftsrats im Bereich der Internationalisierung umzusetzen. Zwar erkennt der Wissenschaftsrat ausdrücklich die vermehrte internationale Ausrichtung der letzten Jahre an und hebt lobend hervor, dass es der Friedens- und Konfliktforschung ,,mit einigen Forschungsschwerpunkten gelungen ist, in internationalen Fachdebatten Themen zu setzen sowie innovative Forschung anzustoßen und voranzutreiben“ (Wissenschaftsrat 2019, S. 11). Gleichzeitig kommt die Mahnung, dass die deutsche Friedens- und Konfliktforschung relativ zu ihrer Ausstattung hinter ihrem Potenzial zurückbleibe. Als konkrete Schritte regt der Wissenschaftsrat eine Erhöhung der Anzahl der in Deutschland tätigen ausländischen Wissenschaftler*innen und eine weitere Steigerung der Veröffentlichungen in international führenden Fachzeitschriften an (ibid.).

Publikationen in führenden internationalen Fachzeitschriften sind für Wissenschaftler*innen im Allgemeinen wichtig, aber vor allem für jüngere Kolleg*innen als wichtigstes Element in der akademischen Karriereplanung unerlässlich. Wie bereits dargestellt, haben laut Wissenschaftsrat vor allem Methoden ,im quantitativen und experimentellen Bereich“ bislang nur unzureichend Eingang in die deutsche Frie- 
dens- und Konfliktforschung gehalten (Wissenschaftsrat 2019, S. 55). Eine breitere Ausbildung in empirisch-analytischen Methoden in Deutschland kann unserer Meinung nach dazu beitragen, die insgesamt noch zu geringe Publikationstätigkeit in international führenden Fachzeitschriften - wovon viele in der empirisch-analytischen Forschung angesiedelt sind - zu verbessern (vgl. Wissenschaftsrat 2019, S. 37). Um hier mit der dynamischen Entwicklung in der internationalen Forschung Schritt zu halten, sind fundierte Methodenkenntnisse für eine gestärkte Internationalisierung daher nicht nur in der Breite erforderlich, um internationale Spitzenforschung „nachvollziehen, einordnen und ggf. kritisch kommentieren zu können“ (Wissenschaftsrat 2019, S. 60), sondern auch in der Tiefe, um selbst zu dieser Forschung beitragen zu können. Eine englischsprachige Abfassung eines Forschungsberichts oder die Einladung von international renommierten Keynote-Speakers zu nationalen Konferenzen (vgl. Engels und Schetter 2020, S. 111) sind sicher einzelne Schritte hin zu einer besseren internationalen Anbindung. Um aber die hiesige Friedens- und Konfliktforschung international sichtbar zu machen, sind, wie der Wissenschaftsrat bemerkt, hochrangige wissenschaftliche Veröffentlichungen der weitaus wichtigere Weg.

Internationale Kooperationen in Form von Verbundprojekten und Gastwissenschaftler*innen werden vom Wissenschaftsrat explizit zu Einrichtungen in Skandinavien, der Schweiz und den USA, ,den derzeit im Forschungsfeld tonangebenden Ländern und Regionen“ (Wissenschaftsrat 2019, S. 48) als wünschenswert erachtet. Auffällig ist bei dieser Auflistung, dass es sich hierbei um Standorte mit ausgeprägten, empirisch-analytischen Methodenkompetenzen handelt. Die im Wissenschaftsbericht erwähnte COST Action ,European Network of Conflict Research" war eine solche Initiative zur europäischen Vernetzung von quantitativen Friedensforscher*innen, von der mehrere Mitglieder des AK Methoden hinsichtlich der methodischen Fortbildung und der internationalen Vernetzung profitiert haben. Eine Zusammenarbeit mit datenintensiven Forschungsprogrammen, wie z.B. dem „Uppsala Conflict Data Program“ (https://ucdp.uu.se) oder der in Zürich ansässigen „Geographical Research on War Unified Platform“ (https://growup.ethz.ch) setzt fortgeschrittene methodische Kenntnisse voraus, um diese Daten adäquat nutzen zu können bzw. sogar selber erhobene Daten beisteuern zu können.

International hochrangige Publikationen kombinieren zunehmend anspruchsvolle, vergleichende Analysemethoden mit hochauflösender Feldforschung in (Post-)Konfliktstaaten (e.g. Fearon et al. 2015; Grossman et al. 2014). Eine Erhöhung der in Deutschland tätigen Wissenschaftler*innen aus Ländern des globalen Südens sowie häufigere Kooperation ,, auf Augenhöhe“ mit Wissenschaftler*innen an Forschungseinrichtungen in diesen Ländern in gemeinsamen Forschungsvorhaben könnten diesen begrüßenswerten Trend weiter fördern und um lokale und regionale Expertise erweitern. Zugleich sollte sichergestellt werden, dass die Ergebnisse dieser Forschung anschließend nicht durch horrende Abonnementgebühren für den Zugang zu Spitzenjournalen einem illustren Kreis westlicher Universitätsangehöriger vorbehalten bleiben. Die durch die Vertreter*innen der deutschen Forschungslandschaft ausgehandelten Open Access Vereinbarungen sind hierzu ein Schritt in die richtige Richtung und sollten auch auf kleinere Verlage mit wichtigen Zeitschriften der Friedens- und Konfliktforschung ausgeweitet werden. 


\subsection{Empirisch-analytische Methoden und deren Relevanz für Politikberatung}

Die momentan zu geringe Breite der methodischen Ansätze in der deutschen Friedens- und Konfliktforschung hat nicht nur Implikationen für den Austausch und die wissenschaftliche Zusammenarbeit innerhalb dieses Forschungsgebiets, sondern auch auf das Zusammenspiel mit Politik, Verwaltung und Gesellschaft. Wie der Wissenschaftsrat anmerkt, ist es eine der Stärken der Friedens- und Konfliktforschung, dass sie die vielfältigen Herausforderungen in einer sich ständig verändernden Welt kritisch beobachten und analysieren kann. Neben der Grundlagenforschung ist daher eine zentrale gesellschaftliche Aufgabe der Friedens- und Konfliktforschung, wissenschaftliche Erkenntnisse für politische Entscheidungsprozesse aufzubereiten und zugänglich zu machen. Wie der Wissenschaftsrat in seiner Evaluation weiter hervorhebt, funktioniert die Interaktion zwischen Wissenschaft und Politik in Deutschland recht gut. Allerdings gibt es auch hier Ausbaupotential im Bereich der evidenzbasierten Friedens- und Konfliktforschung. Diese hat in den vergangenen Jahren beispielsweise große Fortschritte im Bereich von Impact-Studien gemacht, die zunehmend Evaluation konkreter Politikmaßnahmen mit theoriegeleiteter Grundlagenforschung verbindet (vgl. Humphreys und Weinstein 2009). Eine ähnlich dynamische Weiterentwicklung erfährt in den vergangenen Jahren die Bereitstellung hochauflösender Analysen und Daten, die teilweise sogar nahezu in Echtzeit geschieht. ${ }^{3}$

Eine solche systematische Datenbasis aus der empirisch-analytischen Friedensund Konfliktforschung kann eine wichtige zusätzliche Grundlage des politischen Entscheidungsprozesses sein, um z. B. die Entwicklung von politischer Gewalt in der Vergangenheit und der Gegenwart beschreiben zu können. Sie sollte deshalb Teil des Beratungsportfolios sein, welches die Friedens- und Konfliktforschung der Politik zur Verfügung stellt. In einzelnen Projekten hat sich in Deutschland bereits eine gute Zusammenarbeit im Rahmen einer evidenzbasierten Politikberatung entwickelt. ${ }^{4} \mathrm{Im}$ Zeitalter der teils elektronischen Erhebung und somit immer besseren Verfügbarkeit empirischer Daten ist es notwendig, dass die Friedens- und Konfliktforschung in Deutschland in größerem Maße die Kompetenz besitzt, empirische Daten zum Zwecke der Forschung und Politikberatung zusammenzustellen, aufzubereiten, analysieren, interpretieren und teilen zu können. Hierzu bedarf es auch entsprechender Programme zur Identifikation, zur Etablierung und zum Betrieb empirischer Forschungsdateninfrastrukturen in der deutschen Friedens- und Konfliktforschung (vgl. Wissenschaftsrat 2019, S. 57).

Aus diesem Anspruch erwachsen Verpflichtungen, die sich in eine Bring- sowie eine Holschuld einteilen lassen. Eine Erweiterung evidenzbasierter Politikberatung setzt voraus, dass Vertreter*innen evidenzbasierter Forschung abstrakte Ergebnisse so aufbereiten, dass sie über einen engen Expert*innenkreis hinaus verständlich sind. Wie wir selbstkritisch anmerken müssen, ist dies insbesondere in der datenin-

\footnotetext{
3 siehe bspw. https://acleddata.com/ oder https://ucdp.uu.se/.

4 Im Auswärtigen Amt läuft seit einigen Jahren das „PREVIEW“ Projekt zur Krisenfrüherkennung auf der Basis einer Vielzahl empirischer Indikatoren (https://www.auswaertiges-amt.de/de/aussenpolitik/themen/ krisenpraevention/-/2238138). An der Universität der Bundeswehr in München wird unter der Leitung von Carlo Masala ein vergleichbares Projekt durchgeführt.
} 
tensiven Forschung noch nicht ausreichend der Fall. Zugleich besteht jedoch auch eine Holschuld der Politikberatung, da systematische Daten zunehmend als gesichert angesehene Erkenntnisse aus der akademischen, evidenzbasierten Forschung in die Beratungsprozesse einfließen sollten. Dass diese Bring- und Holschuld keine hehren Ziele sind, zeigen die praxisorientierten Publikationen führender Institutionen der Politikberatung (e.g. Friedensgutachten 2020; Bethke 2020; Fiedler und Mross 2019; Haass et al. 2016; Rudolf 2015). Wie auch beim Themenkomplex Interdisziplinarität ist auch hier eine gute und breite Methodenkompetenz wichtig und kann zu einer gestärkten, empirisch fundierten Politikberatung beitragen.

\section{Vernetzung als Chance zur Integration}

Wie können eine breitere Ausbildung und ein besserer Austausch im Bereich Methoden in der deutschen Friedens- und Konfliktforschung erreicht werden? Eine zentrale Empfehlung im Gutachten des Wissenschaftsrats ist die erhöhte Vernetzung der Friedens- und Konfliktforschung in Deutschland. Wie wir oben dargelegt haben, sind eine höhere methodische Diversität und eine Stärkung empirisch-analytischer Methoden Voraussetzung dafür, dass ein solcher Austausch im Dialog mit anderen Disziplinen, aber auch innerhalb der bestehenden scientific community geschehen kann. In den zurückliegenden Jahrzehnten ist die methodische Spezialisierung in allen Schattierungen weit vorangeschritten. Diese schärfere Profilbildung hat ohne Zweifel Bruchlinien entstehen lassen, die auch tief durch die deutsche Friedensund Konfliktforschung laufen. Wir glauben, dass sich diese Trennlinien innerhalb der Wissenschaft und speziell in der AFK als Dachverband überwinden lassen. Thorsten Bonacker (2020) weist treffend darauf hin, dass kooperative Formen der Zusammenarbeit Grenzen des Wettbewerbs überwinden helfen können.

Wie kann eine solche Vernetzung aussehen, um die notwendige methodische Pluralität zu stärken, die der Wissenschaftsrat fordert? Sie besteht zuerst einmal darin, ein Forum zu schaffen, welches den Dialog innerhalb der Forschungsgemeinschaft möglich macht und fördert. Voneinander zu lernen bedeutet, in einen regen Austausch zu treten, benötigt aber auch die Bereitschaft, mit wissenschaftlicher Neugier sich andere Ansätze zu erschließen und neue Kompetenzen zu erwerben. Im Arbeitskreis „Empirische Methoden der Friedens- und Konfliktforschung“, aus dem dieser Beitrag entstand, haben wir versucht, ein solches Forum zu schaffen. Die Mitglieder des Arbeitskreises teilen ihren Fokus auf evidenzbasierte, empirisch-analytische Friedens- und Konfliktforschung, verfolgen diesen aber mit unterschiedlichen Herangehensweisen, die das gesamte methodische Spektrum von qualitativen Einzelfallstudien über ,medium-N“ Studien und Experimente bis hin zu groß-skaligen Vergleichsstudien abdecken. Diese Vernetzung ,im Kleinen“ funktioniert sehr gut, die jährlichen Treffen des AK erfreuen sich großer Beliebtheit. Seit der Gründung des AK im Jahr 2016 findet jedes Jahr ein Workshop mit etwa 40 Teilnehmer*innen aus dem In- und Ausland statt, die Zahl der Einreichungen für diesen Workshop steigt kontinuierlich. Auch der Wissenschaftsrat hebt diese Initiative im Gutachten explizit lobend hervor: „Die Initiative von Konstanzer Wissenschaftlern, die im Rahmen der Arbeitsgemeinschaft für Friedens- und Konfliktforschung einen Arbeitskreis 
Methoden etabliert haben, wird in diesem Zusammenhang ausdrücklich gewürdigt" (Wissenschaftsrat 2019, S. 60). Wir versuchen innerhalb des AK, im gegenseitigen Dialog Brücken zwischen den verschiedenen methodischen Ausrichtungen und Schwerpunkten zu schlagen, die über die verschiedenen Standorte in Deutschland hinweg, aber auch ins Ausland, bestehen. Hier zeigen sich die Vorteile einer überregionalen Vernetzung, da an einzelnen geographischen Standorten oftmals nur wenige empirisch-analytisch arbeitende Forscher*innen vorhanden sind. Aus dieser Erfahrung heraus unterstreichen wir die Empfehlung des Wissenschaftsrats, überregionale Vernetzung zu fördern, um ,eine bessere Verbindung [...] von unterschiedlichen methodischen Zugängen [zu] ermöglichen“ (Wissenschaftsrat 2019, S. 57). Diese Förderung sollte mit einer stärkeren (möglicherweise auch überregionalen) Methodenausbildung verbunden werden, um einzelne Standorte ohne eine ausreichende Repräsentation in empirischen, insbesondere quantitativen Methoden einzubinden. Vor diesem Hintergrund sind die beiden neuen Förderlinien des BMBF zur Stärkung der Friedens- und Konfliktforschung mit dem Fokus auf Regionalzentren, aber eben auch auf überregionale Kompetenznetzwerke ausdrücklich zu begrüßen.

Vernetzung sollte aber nicht nur innerhalb der zurzeit politikwissenschaftlich dominierten Friedens- und Konfliktforschung stattfinden, sondern auch, wie vom Wissenschaftsrat empfohlen, über disziplinäre Grenzen hinweg. Die Zusammenarbeit mit den Wirtschaftswissenschaften und der Soziologie innerhalb des Network of European Peace Scientists sowie des Households in Conflict Networks wurde bereits erwähnt, weitere solcher Initiativen sollten folgen. Ein Bereich, in dem das geschehen sollte, ist beispielsweise die Schnittstelle mit den Kommunikations- und Computerwissenschaften im Bereich digitale Kommunikation. Mobilisierungsprozesse für politische Gewalt spielen sich immer häufiger auf digitalen Kanälen ab und erlauben es der Friedens- und Konfliktforschung, neue und umfangreiche Daten zu digitaler Kommunikation für die Untersuchung dieser Prozesse heranzuziehen. Dafür ist jedoch seitens der Friedens- und Konfliktforschung zumindest Basiswissen über die Erhebung, Verarbeitung und Analyse dieser Daten notwendig. Ähnliches gilt auch für die Kooperation mit der naturwissenschaftlichen Klima(folgen)- und Nachhaltigkeitsforschung, deren komplexe Modellierungsansätze hohe methodische Anforderungen stellen. Um diese Art von Zusammenarbeit möglich zu machen, befürworten wir daher einen stärkeren Fokus auf die Ausbildung in empirisch-analytischer Methodik innerhalb der Friedens- und Konfliktforschung, insbesondere für Studierende, Promovierende und Postdocs, um die interdisziplinäre sowie die internationale Zusammenarbeit zu stärken. Wir sehen diesen Ansatz als einen Baustein unter vielen im methodischen Portfolio der Friedens- und Konfliktforschung in Deutschland. Er hat, wie andere Ansätze auch, bestimmte Stärken und Schwächen und ist für manche Fragestellungen geeignet und für andere nicht. Wir freuen uns, wenn das Gutachten des Wissenschaftsrats als Anlass wirkt, durch eine Stärkung empirisch-analytischer Ansätze das gesamte methodische Potenzial in der deutschen Friedens- und Konfliktforschung besser zu nutzen.

Funding Open Access funding enabled and organized by Projekt DEAL.

Open Access Dieser Artikel wird unter der Creative Commons Namensnennung 4.0 International Lizenz veröffentlicht, welche die Nutzung, Vervielfältigung, Bearbeitung, Verbreitung und Wiedergabe in 
jeglichem Medium und Format erlaubt, sofern Sie den/die ursprünglichen Autor(en) und die Quelle ordnungsgemäß nennen, einen Link zur Creative Commons Lizenz beifügen und angeben, ob Änderungen vorgenommen wurden.

Die in diesem Artikel enthaltenen Bilder und sonstiges Drittmaterial unterliegen ebenfalls der genannten Creative Commons Lizenz, sofern sich aus der Abbildungslegende nichts anderes ergibt. Sofern das betreffende Material nicht unter der genannten Creative Commons Lizenz steht und die betreffende Handlung nicht nach gesetzlichen Vorschriften erlaubt ist, ist für die oben aufgeführten Weiterverwendungen des Materials die Einwilligung des jeweiligen Rechteinhabers einzuholen.

Weitere Details zur Lizenz entnehmen Sie bitte der Lizenzinformation auf http://creativecommons.org/ licenses/by/4.0/deed.de.

\section{Literatur}

Bethke, Felix S. 2020. Gewaltlos zu mehr Demokratie. Wie friedliche Revolutionen nachhaltig demokratische Entwicklung stärken. PRIF Spotlight 7/2020. Frankfurt am Main: Hessische Stiftung für Friedens- und Konfliktforschung. https://www.hsfk.de/fileadmin/HSFK/hsfk_publikationen/ Spotlight0720.pdf. Zugegriffen: 6. Aug. 2020.

BICC Bonn International Center for Conversion, HSFK Leibniz-Institut Hessische Stiftung Friedens- und Konfliktforschung, IFSH Institut für Friedensforschung und Sicherheitspolitik an der Universität Hamburg, und INEF Institut für Entwicklung und Frieden. 2020. Friedensgutachten 2020. Bielefeld: transcript.

Bonacker, Thorsten. 2020. Die Grenzen des Wettbewerbs. Zur Evaluation der Friedens- und Konfliktforschung (nicht nur) aus universitärer Sicht. Zeitschrift für Friedens- und Konfliktforschung 9(1):117-130.

Brühl, Tanja. 2012. Friedensforschung als „Superwissenschaft“ oder Sub-Disziplin? Zum Verhältnis der Friedens- und Konfliktforschung und der Internationalen Beziehungen. Zeitschrift für Internationale Beziehungen 19(1):171-183.

Büttner, Christian. 2004. Friedensforschung - interdisziplinär? Pädagogisch-psychologische Friedensund Konfliktforschung an der Hessischen Stiftung Friedens- und Konfliktforschung. In Friedensund Konfliktforschung in Deutschland, Hrsg. Ulrich Eckern, Leonie Herwartz-Emden, Rainer-Olaf Schulz, und Tanja Zinterer, 219-231. Wiesbaden: VS.

Engels, Bettina, und Conrad Schetter. 2020. Die Gelegenheit nutzen: Die Evaluation der Friedens- und Konfliktforschung durch den Wissenschaftsrat aus der Perspektive der Arbeitsgemeinschaft für Friedens- und Konfliktforschung. Zeitschrift für Friedens- und Konfliktforschung 9(1):105-115.

Fearon, James D., Macartan Humphreys, und Jeremy Weinstein. 2015. How does development assistance affect collective action capacity? Results from a field experiment in post-conflict Liberia. American Political Science Review 109(3):450-469.

Fiedler, Charlotte, und Karina Mross. 2019. Was wir aus der Forschung zu Transitional Justice in Postkonfliktgesellschaften wissen: Wesentliche Erkenntnisse für die Praxis. Bonn: Deutsches Institut für Entwicklungspolitik. https://www.die-gdi.de/uploads/media/AuS_5.2019.pdf. Zugegriffen: 23. Juli 2020.

Gehring, Kai, und Henrik Rubner. 2020. Ein wichtiger Kompass in unruhigen Zeiten. Friedensforschung und die Empfehlungen des Wissenschaftsrates aus Perspektive der Politik. Zeitschrift für Friedensund Konfliktforschung 9(1):167-178.

Grossman, Guy, Macartan Humphreys, und Gabriella Sacramone-Lutz. 2014. "I wld like u WMP to extend electricity 2 our village": On information technology and interest articulation. American Political Science Review 108(3):688-705.

Haass, Felix, Sabine Kurtenbach, und Julia Strasheim. 2016. Flucht vor dem Frieden: Emigration aus Nachkriegsgesellschaften. GIGA Focus Global, Nr. 2. https://www.giga-hamburg.de/de/system/files/ publications/gf_global_1602.pdf. Zugegriffen: 23. Juli 2020.

Humphreys, Macartan, und Jeremy M. Weinstein. 2009. Field experiments and the political economy of development. Annual Review of Political Science 12:367-378.

Niemann, Holger, und Ursula Schröder. 2020. Ein Feld in Bewegung: Friedens- und Konfliktforschung in herausfordernden Zeiten. Zeitschrift für Friedens- und Konfliktforschung 9(1):131-142. 
Reuter, Christian, Jürgen Altmann, Malte Göttsche, und Mirko Himmel. 2020. Zur naturwissenschaftlichtechnischen Friedens- und Konfliktforschung: Aktuelle Herausforderungen und Bewertung der Empfehlungen des Wissenschaftsrats. Zeitschrift für Friedens- und Konfliktforschung 9(1):143-154.

Rudolf, Peter. 2015. Friedensoperationen: Wirksamkeit und Erfolgsbedingungen. Ein Blick auf den Stand der Forschung. SWP-Aktuell 68. https://www.swp-berlin.org/fileadmin/contents/products/aktuell/ 2015A68_rdf.pdf. Zugegriffen: 23. Juli 2020.

Scheffran, Jürgen, und Christiane Fröhlich. 2017. Klima - Gewalt - Flucht: Das Beispiel Syrien. Wissenschaft \& Frieden 2:7-10.

Schneckener, Ulrich, und Thomas Held. 2020. Impulsgeberin und Anstifterin: Die Deutsche Stiftung Friedensforschung im Lichte der Evaluation. Zeitschrift für Friedens- und Konfliktforschung 9(1):155-166.

Verwimp, Philip, Patricia Justino, und Tilman Brück. 2019. The Microeconomics of violent conflict. Journal of Development Economics 141:1-6.

Vollhardt, Johanna Ray, und J. Christopher Cohrs. 2013. Aktuelle sozialpsychologische Beiträge zur Friedens- und Konfliktforschung. Zeitschrift für Friedens- und Konfliktforschung 2(2):246-278.

Wissenschaftsrat. 2019. Empfehlungen zur Weiterentwicklung der Friedens- und Konfliktforschung. Drs., Bd. 7827-19. Gießen: Wissenschaftsrat. 